\title{
Comparative evaluation of Mycobacterium vaccae as a surrogate cloning host for use in the study of mycobacterial genetics
}

\author{
Marco A. Medeiros, ${ }^{1}$ Odir A. Dellagostin, ${ }^{2}$ Geraldo R. G. Armôa, ${ }^{1}$ \\ Wim M. Degrave, ${ }^{3}$ Leila de Mendonça-Lima, ${ }^{3}$ Márcia Q. Lopes, ${ }^{3}$ \\ Joseane F. Costa, ${ }^{3}$ Johnjoe Mcfadden ${ }^{4}$ and Douglas Mclntosh ${ }^{1}$
}

\footnotetext{
1 Laboratory of

Recombinant Technology, Bio-Manguinhos, Oswaldo Cruz Foundation (FIOCRUZ), Rio de Janeiro, Brazil

2 Biotechnology Centre, Federal University of Pelotas, Pelotas, Brazil

3 Department of Biochemistry and Molecular Biology, Oswaldo Cruz Institute, FIOCRUZ, Rio de Janeiro, Brazil

4 School of Biological Sciences, University of Surrey, Guildford, Surrey, UK
}

Author for correspondence: Douglas McIntosh. Tel: +55 212598 4284. Fax: +55 2122604727. e-mail:mcintosh@bio.fiocruz.br

\begin{abstract}
Mycobacterium vaccae represents an alternative mycobacterial cloning host that has been largely overlooked to date. The main reason for this may be the reported non-transformability of this species, specifically the so-called Stanford strain (NCTC 11659), with expression vectors that use kanamycin resistance as a selection method. However, this strain can be transformed using hygromycin resistance as an alternative selectable phenotype. The present study has shown that in contrast to previous reports, M. vaccae (ATCC 15483) is capable of being transformed with a range of vectors encoding kanamycin resistance as the selectable marker. Thereafter, the expression of the lacZ reporter gene in $M$. vaccae, Mycobacterium bovis BCG and Mycobacterium smegmatis $\mathbf{m c}^{2} 155$ was evaluated using a range of characterized mycobacterial promoter sequences (hsp60, hsp70, PAN, 18kDa and $16 S$ rRNA) cloned in the same promoter probe vector. In general, the promoters showed similar levels of activity in the three species, demonstrating that existing expression systems can readily be employed with $M$. vaccae (ATCC 15483). This was further confirmed by the observation that $M$. vaccae was capable of stable, in vitro expression of recombinant $\mathrm{S1}$ subunit of pertussis toxin at levels equivalent to those obtained with BCG and $M$. smegmatis. Analysis of structural and functional stability of a range of vectors demonstrated that the incidence of instability noted for $M$. vaccae was lower than that recorded for $M$. smegmatis. Taken together, the results indicate that $M$. vaccae is an additional cloning host which may prove useful for specific aspects of mycobacterial biology and provide increased flexibility to the field of recombinant protein technology for mycobacteria.
\end{abstract}

Keywords: mycobacteria, shuttle vectors, expression, stability

\section{INTRODUCTION}

Surrogate cloning hosts have played an important role in developing our knowledge of mycobacterial genetics by allowing the functional analysis of genes and regulatory sequences from both rapid growing species (Mahenthiralingam et al., 1993; Parish et al., 1997) and the slow or non-cultivable pathogenic members of this genus, including Mycobacterium tuberculosis, Mycobacterium leprae and members of the Mycobacterium avium/Mycobacterium intracellulare complex (Hermans \& de Bont 1996; Jacobs, 2000).
Initial surrogate cloning hosts included Escherichia coli and Streptomyces lividans (Clarke-Curtiss et al., 1985; Kieser et al., 1986). However, it was only following the discovery of a transformation efficient (ept) mutant strain of the rapid growing species Mycobacterium smegmatis, i.e. strain $\mathrm{mc}^{2} 155$ (Snapper et al., 1990), that the cloning and study of genes from pathogenic and non-pathogenic mycobacteria became a relatively common practice. The utility of this bacterium as a cloning host is well established, and its characteristics and applications to the study of mycobacterial genetics have recently been reviewed (Jacobs, 2000). 
Certainly, the possible use of other species of mycobacteria as cloning hosts has received some attention (Parish \& Stoker, 1997; Hermans \& de Bont, 1996). In particular, Mycobacterium aurum has been employed in a number of studies (Hermans et al., 1990, 1993; Lazraq et al., 1991). This species was considered to be attractive for the study of the genetic mechanisms involved in sensitivity and resistance to the antibiotics used in the treatment of both tuberculosis and leprosy (Lazraq et al., 1991).

In addition, Mycobacterium vaccae, specifically the socalled Stanford strain (NCTC 11659), has been employed as a cloning host for the expression of recombinant forms of mycobacterial proteins including superoxide dismutase (Garbe et al., 1994), major extracellular proteins (Harth et al., 1997) and the $19 \mathrm{kDa}$ lipoprotein of M. tuberculosis (Abou-Zeid et al., 1997), and for the development of an epitope delivery system for use with recombinant mycobacteria (Hetzel et al., 1998). Interestingly, Golanska et al.(1998) reported a biotechnological application for M. vaccae, where the mutant B3805 was used for cloning and expression of genes involved in steroid degradation pathways. This species has also attracted interest for use in immunotherapy of tuberculosis (Corlan et al., 1997; Onyebujoh et al., 1995).

A review of the literature concerning transformation of mycobacteria reveals that the overwhelming majority of vectors used with this genus contain a gene encoding resistance to kanamycin as a selectable marker (Pashley \& Stoker, 2000; Parish \& Stoker, 1997; Hermans \& de Bont, 1996). However, some species including M. vaccae (NCTC 11659) have been reported to be non-transformable using this selection method, possibly due to inefficient expression of the aminoglycoside phosphotransferase gene involved in kanamycin resistance (Garbe et al., 1994). Consequently, vector systems containing a gene encoding resistance to hygromycin have been developed that allow the transformation of this species (Garbe et al., 1994; Parish \& Stoker, 1997). Hygromycin resistance is considered attractive in providing a second selectable marker for genetic experiments with mycobacteria and in providing a resistance marker that avoids the use of a clinically applicable drug (Garbe et al., 1994). Yet, the use of hygromycin instead of kanamycin suffers from a number of disadvantages including expense (hygromycin is approximately 15 times more expensive than kanamycin), low-level mammalian toxicity and the need to use elevated concentrations of this antibiotic to select E. coli transformants (200 $\mu \mathrm{g} \mathrm{ml}^{-1}$ instead of $20 \mu \mathrm{g} \mathrm{ml}^{-1}$ for kanamycin). Finally, and perhaps most significantly, almost all available mycobacterial vectors contain a kanamycin resistance gene. The possibility exists that these factors may have contributed to the relatively limited interest shown for the use of M. vaccae as a surrogate cloning host, despite indications that it may represent a valuable alternative to the more commonly employed M. smegmatis $\mathrm{mc}^{2} 155$.

In this report we describe the successful transformation of M. vaccae (ATCC 15483) with a range of vectors encoding resistance to kanamycin as a selectable marker. Thereafter, our objective was to extend this observation through a comparative evaluation of this strain of $M$. vaccae and $M$. smegmatis $\mathrm{mc}^{2} 155$ as cloning hosts, with emphasis on the functional activity of promoter sequences used in expression vectors, the production of heterologous proteins and stability (both functional and structural) of vector constructs.

\section{METHODS}

Bacterial strains and plasmids. M. bovis BCG (Moreau strain), M. smegmatis $\mathrm{mc}^{2} 155$ and $M$. vaccae ATCC 15483, were obtained from the mycobacteria collection of The Oswaldo Cruz Institute (Rio de Janeiro, Brazil). All transformations in E. coli were performed with strain DH5 $\alpha$. M. bovis BCG was grown in Middlebrook 7H9 broth (Difco) supplemented with $10 \%$ albumin/glucose (ADC), $0 \cdot 2 \%$ glycerol and $0.05 \%$ Tween 80 (Sigma), or 7H10 agar (Difco) containing 10\% albumin/glucose (ADC) and $0 \cdot 2 \%$ glycerol. E. coli was cultured in Luria-Bertani (LB) broth or the same medium solidified with $1.5 \%$ agar. M. smegmatis and M. vaccae were cultured in LB broth supplemented with $0.05 \%$ Tween 80 or on plates of LB agar. When necessary, media were supplemented with hygromycin B at $50 \mu \mathrm{g} \mathrm{ml}^{-1}$ (for mycobacteria) or $200 \mu \mathrm{g} \mathrm{ml}^{-1}$ (for E. coli), or $25 \mu \mathrm{g}$ kanamycin $\mathrm{ml}^{-1}$ for

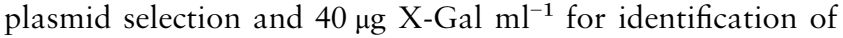
recombinant clones with $\beta$-galactosidase activity. The $E$. coli/mycobacteria shuttle vectors pJEM11 (Lim et al., 1995) and pJEM15 (Timm et al., 1994) were kindly provided by Dr Brigitte Gicquel, Institute Pasteur, Paris, France. The vector pMV261 (Stover et al., 1991) was a gift from Dr A. Cataldi (Instituto de Biotecnologia, INTA, Hurlingham, Argentina) and $\mathrm{pBEN}$, a derivative of pMV262 expressing a mutated form of green fluorescent protein (Cormack et al., 1996) under control of the $h s p 60$ promoter, was generously supplied by $\mathrm{Dr}$ L. Ramakrishnan (Stanford University, USA).

Plasmid construction. A schematic representation of the steps involved in the design of the pUS series of vectors is provided in Fig. 1. Plasmid pUS971 was constructed by cloning a $1.6 \mathrm{~kb}$ PCR fragment from pAL5000, corresponding to nt 4311-1100 and containing the mycobacterial origin of replication (oriM), into pUC18 digested with EcoRI/AflIII and treated with the Klenow fragment of DNA polymerase I. This construct was subsequently digested with BmpI/HindIII to excise the ampicillin resistance gene and PCR-generated DNA fragments representing aph (kanamycin resistance gene) from Tn903 or the hygromycin resistance gene of Streptomyces hygroscopicus were cloned in its place to produce pUS972 and pUS982 respectively. The vector pUS972 contains a kanamycin resistance marker, includes both an E. coli and mycobacterial origin of replication and a multiple cloning site, and formed the basis for the production of all subsequent plasmids of the pUS series developed in this study. Sequences corresponding to the $h s p 60$ promoter of $M$. bovis BCG and the PAN promoter derived from IS900 of Mycobacterium paratuberculosis were generated by PCR, digested with BamHI/KpnI then cloned into pUS972 cut with the same enzymes, giving rise to two expression vectors i.e. pUS973 (Phsp60) and pUS977 (PAN), suitable for the production of recombinant proteins. A promoter-probe vector, pUS986, was developed by cloning a 3083 bp PCR-generated fragment, digested with HindIII, of the E. coli lacZ gene sequence derived from pMC1871, into HindIII-digested pUS972. This vector allows quantitative assessment of promoter activity in vitro based on the measurement of $\beta$-galactosidase activity derived from the 

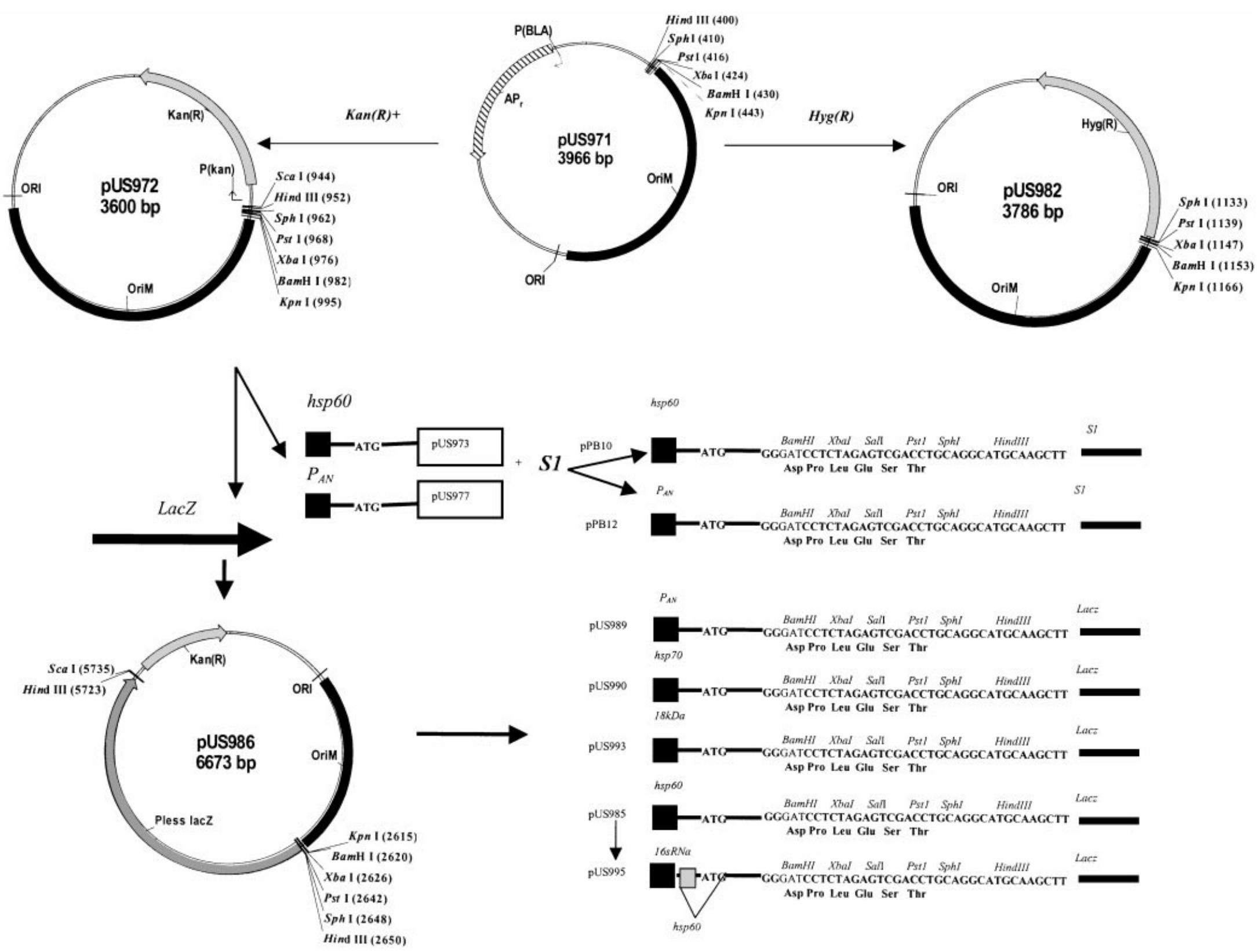
Table 1. Primers used in this study

\begin{tabular}{|c|c|c|c|}
\hline Amplicon & & Primer sequence $\left(5^{\prime}-3^{\prime}\right)$ and orientation & Template \\
\hline \multirow{2}{*}{$\begin{array}{l}\text { S1 subunit pertussis toxin } \\
(815 \mathrm{bp})\end{array}$} & $\mathrm{F}$ & GGGAATTCAAGCTTGCGTTGCACTCGGCA & Bordetella pertussis genomic DNA \\
\hline & $\mathrm{R}$ & GGGAATTCAACGCTTCTAGAACGAATACGCG & \\
\hline \multirow[t]{2}{*}{ Phsp60 (384 bp) } & $\mathrm{F}$ & TCGGTACCCCGACCACAACGAC & M. bovis BCG genomic DNA \\
\hline & $\mathrm{R}$ & GAGGATCCCCCGTCTTGGCCAT & \\
\hline \multirow[t]{2}{*}{ Phsp70 (160 bp) } & $\mathrm{F}$ & GAGGTACCACCCGCACGACCAG & M. tuberculosis genomic DNA \\
\hline & $\mathrm{R}$ & CGGGATCCACCGCACGAGCCATG & \\
\hline \multirow[t]{2}{*}{ PAN (159 bp) } & $\mathrm{F}$ & TCCGGTACCAAGGCCGAAGAG & M. paratuberculosis genomic DNA \\
\hline & $\mathrm{R}$ & CGGGATCCCTTGACAACGTCA & \\
\hline \multirow[t]{2}{*}{$\mathrm{P} 18 k D a(263 \mathrm{bp})$} & $\mathrm{F}$ & AGGGTACCGCAGCGACGGCAC & M. leprae genomic DNA \\
\hline & $\mathrm{R}$ & CAGGATCCATCAGCATGTGTGG & \\
\hline \multirow[t]{2}{*}{ P16S RNA (179 bp) } & $\mathrm{F}$ & TCGGTACCTATGGATATCTATG & M. tuberculosis genomic DNA \\
\hline & $\mathrm{R}$ & GGGGTCACCAAGAGCATGGCCA & \\
\hline \multirow[t]{2}{*}{ lacZ (3083 bp) } & $\mathrm{F}$ & CCCAAGCTTGGTCGTTTTACAACGTCG & Plasmid pMC1871 \\
\hline & $\mathrm{R}$ & CCCAAGCTTGCCCCTGCCCGGTTATTA & \\
\hline \multirow[t]{2}{*}{ OriM (1629 bp) } & $\mathrm{F}$ & CCCAGCCCACCAGCTCCGTAAG & Plasmid pAL5000 \\
\hline & $\mathrm{R}$ & CCCGACACCCGCTCCCCAATTG & \\
\hline \multirow[t]{2}{*}{ Hyg (1122 bp) } & $\mathrm{F}$ & CTGTAAAGCCTGGGGTGCCTAAT & Plasmid pOLYG \\
\hline & $\mathrm{R}$ & GTGCCTCACTGATTAAGCATTG & \\
\hline
\end{tabular}

promoterless lac $Z$ sequence. To generate promoter-containing derivatives of pUS986, DNA fragments corresponding to the hsp60 promoter of $M$. bovis BCG, the hsp70 and the $18 \mathrm{kDa}$ promoter of $M$. leprae and the PAN promoter were produced by PCR, digested with BamHI/KpnI and subsequently cloned into pUS986 cut with the same restriction endonucleases. This procedure resulted in the vectors pUS985, pUS989, pUS990 and pUS993. A fifth vector (pUS995) was produced by digestion of the vector pUS985 with BamHI/BstEII and subsequent introduction of a PCR-derived amplicon, digested using the same enzymes and representing the $16 S$ rRNA promoter of M. tuberculosis, designed to replace the -35 and -10 regions of the $h s p 60$ promoter while leaving the ribosome binding site and start codon of the $h s p 60$ promoter in place. These constructs allowed a meaningful comparison of the relative strengths of different promoters within the same vector.

To construct pPB10 and pPB12, a 815 bp fragment of the gene encoding the $S 1$ subunit of pertussis toxin was amplified by PCR using Bordetella pertussis genomic DNA as the template. The resulting amplicon was digested with HindIII and cloned into pUS973 or pUS977 as appropriate. Details of the primers used in the above-mentioned procedures are provided in Table 1.

Transformation of mycobacteria. M. smegmatis $\mathrm{mc}^{2} 155$ and M. vaccae were grown in $5 \mathrm{ml} \mathrm{LB}$ broth containing $0.05 \%$ Tween 80 for 72 and 96 h respectively, to produce saturated starter cultures. Starter cultures were diluted 100 -fold in $100 \mathrm{ml}$ of the same growth medium in $500 \mathrm{ml}$ conical flasks and were grown with shaking at $37^{\circ} \mathrm{C}$ for $48 \mathrm{~h}$ (M. smegmatis) or $72 \mathrm{~h}$ (M. vaccae). Cultures of $M$. bovis BCG for electroporation were produced in a similar manner using $7 \mathrm{H} 9$ broth as the growth medium. Here, starter cultures were produced over 14 days and the working cultures were grown for $10 \mathrm{~d}$ with shaking at $37^{\circ} \mathrm{C}$. Cultures of M. smegmatis and M. vaccae were incubated on ice for $1.5 \mathrm{~h}$ prior to harvesting by centrifugation at $3000 \mathrm{~g}$ for $10 \mathrm{~min}$ at $4{ }^{\circ} \mathrm{C}$, washed three times with ice cold $10 \%$ glycerol and finally resuspended in
$1 \mathrm{ml} 10 \%$ glycerol. In the case of M. bovis BCG, cultures were held at ambient temperature $\left(25^{\circ} \mathrm{C}\right)$ during harvesting and all subsequent washing stages. In some experiments, glycine (final concentration $1.5 \%$ ) was added to cultures during the last $24 \mathrm{~h}$ of growth. In others, the influence of elevated temperature on transformation efficiencies was examined by omitting the pre-chilling stage and performing all washes at ambient temperature. Electroporation was performed using standard methods (Parish \& Stoker, 1997). Briefly, $100 \mu \mathrm{l}$ electrocompetent cells were mixed with $0 \cdot 05-3 \mu \mathrm{g}$ plasmid DNA and transformed in $0.2 \mathrm{~cm}$ electroporation cuvettes, using a Bio-Rad Gene Pulser set at $2.5 \mathrm{kV}, 25 \mu \mathrm{F}$ and $1000 \Omega$. Cells were immediately diluted with $0.9 \mathrm{ml}$ of the appropriate growth medium without antibiotics and were incubated for $4 \mathrm{~h}$ (M. smegmatis and M. vaccae) or $16 \mathrm{~h}$ (M. bovis BCG) at $37^{\circ} \mathrm{C}$ before plating on LB or $7 \mathrm{H} 10$ agar containing the appropriate antibiotic and/or X-Gal. Transformants were selected after 3 to 7 days (M. smegmatis and M.vaccae) and 14 to 21 days (M. bovis BCG).

$\boldsymbol{\beta}$-Galactosidase assay. $\beta$-galactosidase activity was assayed in E. coli and mycobacteria as described by Miller (1992). Duplicate $1 \mathrm{ml}$ volumes of test cultures $\left(\mathrm{OD}_{600} 0 \cdot 8-1 \cdot 2\right)$ were collected by centrifugation in $2 \mathrm{ml}$ screw cap microcentrifuge tubes (Bio-Rad), the supernatant was removed by aspiration and $500 \mu \mathrm{l}$ glass beads $(0 \cdot 1 \mathrm{~mm}$ diameter; Biospec Products) and $1 \mathrm{ml} \mathrm{Z}$ buffer (Miller, 1992) were added to each tube. Cells were disrupted on a mini beadbeater apparatus (Biospec Products) by mixing for 1 min or $1.5 \mathrm{~min}$ (M. bovis BCG) with $30 \mathrm{~s}$ pulses, with tubes held on ice before and between pulses. After separation of the beads by centrifugation, aliquots $(0.5 \mathrm{ml})$ of the resulting supernatants were transferred to $1.5 \mathrm{ml}$ microcentrifuge tubes and held at $28{ }^{\circ} \mathrm{C}$ for $5 \mathrm{~min}$.

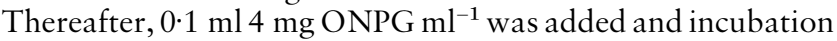
was continued at $28^{\circ} \mathrm{C}$ until sufficient yellow colour had developed, at which point the reaction was stopped by adding $250 \mu \mathrm{l} 1 \mathrm{M} \mathrm{Na}_{2} \mathrm{CO}_{3}$ and the incubation time was recorded. The $\mathrm{OD}_{420}$ of each reaction mix was measured and $\beta$ galactosidase activity was calculated using the formula reported by Dellagostin et al. (1995). 
Expression and detection of recombinant S1 subunit of pertussis toxin in mycobacteria. Mycobacteria transformed with the vectors pUS973, pUS977, pPB10 and pPB12 were grown to a density of approximately $10^{8}$ cells $\mathrm{ml}^{-1}$ in liquid medium containing $25 \mu \mathrm{g}$ kanamycin $\mathrm{ml}^{-1}$. Volumes $(2 \mathrm{ml})$ of each culture were sedimented at $10000 \mathrm{~g}$ in $2 \mathrm{ml}$ screw cap microcentrifuge tubes and prepared for Western blotting analysis by washing once in PBS, followed by resuspension in $100 \mu \mathrm{l}$ lysis buffer $(10 \mathrm{mM}$ Tris/ $\mathrm{HCl}, \mathrm{pH} 7 \cdot 5,1 \mathrm{mM}$ EDTA, $2 \mathrm{mM}$ PMSF) in the presence of $50 \mathrm{mg}$ glass beads $(0.1 \mathrm{~mm}$ diameter). Cells were disrupted by two $30 \mathrm{~s}$ pulses in a mini beadbeater, mixed with $33 \mu \mathrm{l} 4 \times$ concentrated SDS-PAGE sample buffer (Laemmli, 1970) then boiled for $10 \mathrm{~min}$. The beads were allowed to settle and samples of the uncleared cell lysate were analysed by SDS-PAGE and Western blots (Towbin, 1979). The anti-S1 monoclonal antibody (3CX4PT, kindly donated by Dr Drusilla Burns, Food and Drug Administration, Bethesda, MD, USA) was employed at a dilution of 1:6000 and protein bands reacting with this antibody were visualized using alkaline phosphatase conjugated goat anti-mouse IgG (Sigma), with subsequent incubation with BCIP/NBT (5-bromo-4-chloro-3-indolyl phosphate/nitro blue tetrazolium) for colour development.

Shuttle vector stability in mycobacteria and E. coli. Mycobacterial transformants were cultured in liquid medium containing the appropriate antibiotic over three consecutive subcultures, while E. coli transformants were grown over 10 consecutive subcultures, diluting cultures $1 / 1000$ in fresh medium each time. Total DNA was collected from the mycobacterial cultures using the small scale method reported by Santos et al. (1992). Plasmid DNA was produced from E. coli using the Flexiprep kit (Amersham Pharmacia-Biotech), according to the manufacturer's instructions. Electrocompetent cells of $E$. coli $\mathrm{DH} 5 \alpha$ were transformed by electroporation with $2 \mu$ l total mycobacterial DNA and were plated on LB agar containing $25 \mu \mathrm{g}$ kanamycin or $200 \mu \mathrm{g}$ hygromycin $\mathrm{B} \mathrm{ml} \mathrm{m}^{-1}$ as appropriate. Three individual colonies from each transformation were collected and used to produce plasmid DNA that was digested with the restriction endonucleases BamHI, EcoRI and HindIII (Gibco BRL). Electrophoresis of digested plasmids was performed in $1 \%$ agarose gels and banding patterns were visualized with ethidium bromide under UV illumination. The presence of gross structural modifications was assessed by comparison with the banding patterns generated from the plasmid preparations used to produce the original transformants.

\section{RESULTS AND DISCUSSION}

Previous studies have indicated that shuttle vectors that encode resistance to kanamycin are unsuitable for use with M. vaccae (NCTC 11659). However, data from initial experiments showed that an alternative strain of this species, ATCC 15483, could be transformed with such vectors. Based on this observation, a comparative study of the transformability of $\mathrm{M}$. vaccae (ATCC 15483 ) and M. smegmatis $\mathrm{mc}^{2} 155$ was performed. We employed previously characterized vectors including pJEM15 (Timm et al., 1994), pJEM11 (Lim et al., 1995) pMV261 (Stover et al., 1991) and pBEN, a derivative of pMV262 expressing a mutated form of green fluorescent protein (Cormack et al., 1996). In addition, a novel series of plasmids (pUS series) was developed and evaluated. The pUS series comprises two basic shuttle vectors pUS972 (encoding kanamycin resistance) and
Table 2. Representative efficiency of transformation by electroporation of $M$. bovis BCG (Moreau), $M$. smegmatis $\mathrm{mc}^{2} 155$ and $M$. vaccae

Transformation efficiencies were calculated as the mean number of kanamycin or hygromycin-resistant colonies recovered from duplicate $100 \mu \mathrm{l}$ samples of electrocompetent cells using a standard quantity of $1 \mu \mathrm{g}$ DNA. Equivalent transformation efficiencies were obtained using $0 \cdot 1 \mu \mathrm{g}$ DNA. Lower quantities of DNA resulted in reduced transformation efficiencies and quantities greater than $1 \mu \mathrm{g}$ did not increase transformation efficiencies. Each experiment was repeated three times using different batches of freshly prepared

electrocompetent cells. Intra-experiment variations of as much as 10 -fold were observed between different batches of cells, but the differential efficiency of the test vectors was observed in all experiments. NT, Not tested.

\begin{tabular}{|lccc|}
\hline Vector & M. bovis BCG & $\begin{array}{c}\text { M. smegmatis } \\
\text { mc }^{2} \text { 155 }\end{array}$ & M. vaccae \\
\hline pJEM15 & $6 \cdot 1 \times 10^{2}$ & $8 \cdot 4 \times 10^{3}$ & $5 \cdot 2 \times 10^{3}$ \\
pJEM11 & $5 \cdot 4 \times 10^{2}$ & $6 \cdot 2 \times 10^{3}$ & $1 \cdot 0 \times 10^{4}$ \\
pUS972 & $8 \cdot 2 \times 10^{2}$ & $2 \cdot 5 \times 10^{4}$ & $9 \cdot 0 \times 10^{3}$ \\
pUS982 & $3 \cdot 0 \times 10^{2}$ & $1 \cdot 7 \times 10^{4}$ & $1 \cdot 3 \times 10^{5}$ \\
pUS986 & $4 \cdot 6 \times 10^{2}$ & $4 \cdot 3 \times 10^{4}$ & $6 \cdot 5 \times 10^{3}$ \\
pMV261 & $\mathrm{NT}$ & $\mathrm{NT}$ & $4 \cdot 2 \times 10^{3}$ \\
pBEN & $\mathrm{NT}$ & $\mathrm{NT}$ & $2 \cdot 9 \times 10^{3}$ \\
\hline
\end{tabular}

pUS982 (encoding hygromycin resistance), a promoter probe vector pUS986 (containing a promoterless lacZ gene) and a range of expression vectors based on pUS972 which incorporate a range of previously characterized mycobacterial promoter sequences.

\section{Transformation of mycobacteria by electroporation}

Details of representative transformation efficiencies obtained using the test vectors for the transformation of M. bovis BCG, M. smegmatis $\mathrm{mc}^{2} 155$ and $M$. vaccae ATCC 15483 by electroporation are provided in Table 2 , along with values obtained using $M$. vaccae and the vectors pMV261 and pBEN. Transformation efficiencies were calculated as the number of antibiotic resistant colonies recovered from $100 \mu$ lelectrocompetent cells per $\mu$ g vector DNA. It was observed that all three species could be transformed with each of the vectors examined, with the highest transformation efficiencies being recorded for M. smegmatis. An exception to this was the vector pUS982 and M. vaccae, where the level of transformation was 10 -fold higher than that observed for $\mathrm{mc}^{2} 155$ with the same vector. The pre-treatment of cultures with glycine (final concentration $1.5 \%$ during the last $24 \mathrm{~h}$ growth) did not have a marked effect upon the observed efficiency of transformation for any species with any vector (data not shown). In contrast, the use of an elevated temperature $\left(25^{\circ} \mathrm{C}\right)$, instead of the more commonly used $4{ }^{\circ} \mathrm{C}$ for the preparation of cells for the electroporation process was found to greatly reduce the number of transformants of both M. smegmatis and $M$. vaccae for all of the vectors examined (data not shown). 
Table 3. Comparison of the activities of characterized mycobacterial promoters in different cloning hosts

$\beta$-Galactosidase activity was calculated using the formula $\left(\mathrm{OD}_{420} \times 1000\right) /\left(t \times v \times \mathrm{OD}_{600}\right)$ where $t$ is the incubation time in min and $v$ is the volume in millilitres of culture used. The values shown are the mean activities resulting from two independent experiments each employing two individual clones, which were processed and assayed in duplicate.

\begin{tabular}{|c|c|c|c|c|c|}
\hline \multirow[t]{2}{*}{ Vector/promoter } & \multirow[b]{2}{*}{ Cloning host... } & \multicolumn{4}{|c|}{$\beta$-Galactosidase activity (Miller $\mathrm{U} \pm \mathrm{SD}$ ) } \\
\hline & & M. bovis BCG & M. smegmatis & M. vaccae & E. coli \\
\hline pUS986 (vector) & & $2 \cdot 5$ & $3 \cdot 0$ & $2 \cdot 0$ & $8 \cdot 0$ \\
\hline pUS985 (hsp60) & & $6391 \pm 551$ & $1131 \pm 108$ & $2254 \pm 75$ & $220 \pm 10$ \\
\hline pUS989 $\left(P_{\mathrm{AN}}\right)$ & & $1375 \pm 80$ & $2592 \pm 166$ & $6443 \pm 301$ & $11 \cdot 8 \pm 2 \cdot 5$ \\
\hline pUS990 (hsp70) & & $1130 \pm 61$ & $1436 \pm 50$ & $1724 \pm 63$ & $227 \pm 17$ \\
\hline pUS993 (18kDa) & & $1259 \pm 127$ & $1715 \pm 116$ & $3305 \pm 188$ & $11310 \pm 849$ \\
\hline pUS995 (16S RNA) & & $113 \pm 14$ & $44 \pm 4$ & $293 \pm 17$ & $1077 \pm 76$ \\
\hline
\end{tabular}

The vectors from the pUS series are similar to the majority of other published, replicative mycobacteria-E. coli shuttle vectors, but differ owing to the reduced size of the mycobacterial origin of replication used in their construction. However, this factor alone cannot explain the apparent dichotomy in transformability of different strains of $M$. vaccae, given that we were able to transform this species with the vectors pJEM11, pJEM15, pMV261 and pBEN. In common with the vectors pBAK14, pYUB12 and pMV251 employed by Garbe et al. (1994), the above-mentioned plasmids all contain fragments of pAL5000 that are larger than that present in the pUS series of vectors. An alternative explanation could be that there exist some subtle differences between the NCTC 11659 and the ATCC 15483 strains of $M$. vaccae, which may be responsible for the reported inability to transform the former strain with vectors encoding resistance to kanamycin. This possibility is strengthened by the fact that the ATCC strain could be transformed with two representatives of the pMV vector series (Stover et al., 1991), while the NCTC strain was found to be non-transformable with pMV251 (Garbe et al., 1994), a vector from the same series which contains an identical aph gene and pAL5000 sequences to those present in the pMV plasmids used in the present study.

In the study of Garbe et al. (1994), data provided for transformation efficiencies were based on a comparison of the vectors pBAK14 (kan) and p16R1 (byg). These vectors differed not only in terms of their selectable marker, but also with respect to size and the composition of their basic structure. As such, it is not clear if the resistance gene component was the true reason behind the inability of the pBAK14 vector to transform $M$. vaccae NCTC 11659 . In an attempt to clarify this issue, we performed a comparison of the vectors pUS972 and pUS982, which are essentially identical in all aspects except their resistance marker. This experiment revealed that the presence of the $b y g$ sequence resulted in a vector with an improved capacity to transform $M$. vaccae. Indeed, this vector produced the highest transformation efficiencies recorded in this study and an argument could be made for using hygromycin resistance as the marker of choice with M. vaccae. However, it should be emphasized that the efficiencies recorded with the other vectors are more than sufficient for most applications. Furthermore, in contrast to the observations of Garbe at al. (1994), we found the vectors conferring kanamycin resistance to be superior to pUS982 in terms of the efficiency of transformation achieved with BCG and $M$. smegmatis $\mathrm{mc}^{2} 155$, and we did not detect any marked difference in the growth rate of the two types of transformants.

\section{Comparison of promoter activity in different cloning hosts}

The ability to readily transform $M$. vaccae with previously described cloning vectors and with those developed in this study was considered a significant observation. Therefore, experiments were performed to evaluate the suitability of this species as a cloning host in comparison with $M$. smegmatis $\mathrm{mc}^{2} 155$. Initial experiments examined the functional activity of a range of previously characterized mycobacterial promoters in three mycobacterial cloning hosts and in E. coli. The promoters used in the present study have been employed by other workers for the development of mycobacterial expression vectors or in the study of gene regulation in mycobacteria (Murray et al., 1992; Das Gupta et al., 1993; Yasutomi et al., 1993; Dellagostin et al., 1995). Yet, to our knowledge no study has compared the functional activities of these promoters when cloned in a unique expression system and there is no information on their ability to regulate gene expression in M. vaccae.

The promoters used were the $h s p 60$ promoter of $M$. bovis BCG, the $h s p 70$ and the $18 \mathrm{kDa}$ promoters of $M$. leprae, the PAN promoter of M. paratuberculosis and the $16 S r R N A$ promoter of $M$. tuberculosis. The vectors pUS986 (promoterless), 985 (hsp60), 989 (PAN), 990 (hsp70), $993(18 k D a)$ and 995 (16S rRNA) were used to transform $M$. bovis BCG, M. smegmatis $\mathrm{mc}^{2} 155, M$. vaccae ATCC 15483 and E. coli by electroporation. The transformation efficiencies recorded for these vectors 
were similar to those reported in Table 2, with the exception of pUS985 which routinely gave rise to 10- to 100-fold fewer transformants than the other constructs, this effect being most noticeable in $M$. smegmatis $\mathrm{mc}^{2} 155$.

Functional activities (Miller $\mathrm{U} \beta$-galactosidase activity) of the test promoters in the three species of mycobacteria and in E. coli are shown in Table 3. All promoters showed activity in the three species of mycobacteria, although the relative strengths varied as a function of the species in which they were cloned. Thus, the $h s p 60$ promoter of $M$. bovis was seen to be the strongest promoter in BCG where it was approximately five times more active than the $h s p 70, P$ AN and $18 k D a$ promoters, and 56 times more active than the $16 S r R N A$ promoter of M. tuberculosis (Table 3). In M. smegmatis $\mathrm{mc}^{2} 155$, the $h s p 60, h s p 70$ and $18 k D a$ promoters exhibited similar levels of activity which were approximately fourfold lower than that of the hsp 60 promoter in BCG (Table 3). The PAN promoter was found to be the strongest promoter in this species of mycobacteria, where it showed almost twice the level of activity recorded in BCG. In the case of M. vaccae, the PAN promoter was also found to have the highest activity among the test promoters, showing almost three times the activity of the two heat-shock gene promoters and approximately two times more activity than the $18 k D a$ promoter (Table 3). In common with the situation in BCG, the $16 \mathrm{~S} r R N A$ promoter was found to function relatively poorly in both M. smegmatis and M. vaccae.

The relatively high activity of the $18 k D a$ promoter, contained in pUS993, in all three mycobacterial species was an unexpected result since this promoter has previously been shown to have very low in vitro activity in both $M$. smegmatis $\mathrm{mc}^{2} 155$ and BCG (Glaxo) transformed with the replicative vectors pUS935 and pUS936 (Dellagostin et al., 1995). It should be noted however, that these constructs differed from pUS993 both in terms of size and with regard to the precise nature of their promoter-containing region.

Data obtained with E. coli revealed that the majority of the test promoters functioned poorly in this genetic background. A notable exception was the $18 k D a$ promoter of M. leprae which showed the highest activity (11310 Miller U) for any promoter in any cloning host examined (Table 3). Similarly, the $16 S r R N A$ promoter was found to function optimally in E. coli, where it was almost four times as strong as in M. vaccae and 25 times more active than in $M$. smegmatis $\mathrm{mc}^{2} 155$. The relatively low activity in mycobacteria was surprising in the light of previously reported high activity of this promoter in both BCG (Glaxo) and M. smegmatis LR222, when cloned in the integration-proficient expression vector pDK20 (Das Gupta et al., 1998).

The experiments investigating the functional activity of promoters in the different cloning hosts were performed with the objective of determining whether existing mycobacterial expression systems could be employed

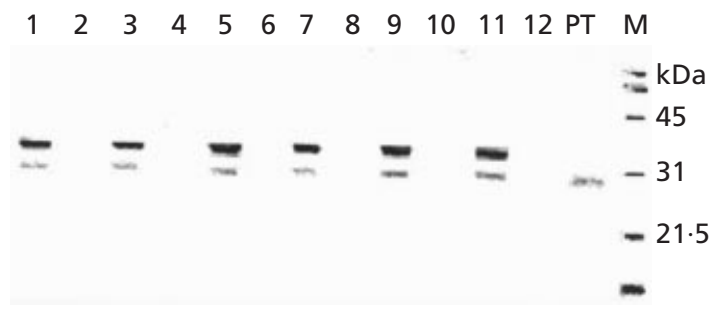

Fig. 2. Immunoblot detection of $\mathrm{S} 1$ subunit of pertussis toxin expression by recombinant $M$. bovis BCG, $M$. smegmatis $\mathrm{mc}^{2} 155$ and $M$. vaccae. Lanes: 1, BCG(pPB10); 2, BCG(pUS973); 3, BCG(pPB12); 4, BCG(pUS977); 5, $\mathrm{mc}^{2} 155$ (pPB10); 6, $\mathrm{mc}^{2} 155$ (pUS973); 7, $\mathrm{mc}^{2} 155$ (pPB12); 8, $\mathrm{mc}^{2} 155$ (pUS977); 9, $M$. vaccae(pPB10); $10, M$. vaccae(pUS973); $11, M$. vaccae(pPB12); 12 , M. vaccae(pUS977). PT, pertussis toxin (40 ng); M, molecular mass standards.

with M. vaccae and this was found to be the case. However, it should be noted that no attempt was made to investigate the possible influence that differences in plasmid copy number may have exerted upon the levels of $\beta$-galactosidase activity recorded in the three species. In the absence of such data it is not possible to conclusively state that the differences in enzyme activity observed with some promoters were solely the result of differences in promoter function in a given host. Previous studies have indicated that pAL5000-based vectors are present at between 2 and 5 copies in M. smegmatis (Pashley \& Stoker, 2000; Stolt \& Stoker, 1996); it would be of value for future studies to address this issue of plasmid biology in other cloning hosts including BCG and M. vaccae.

\section{Cloning and expression of the $\mathbf{S} 1$ subunit of pertussis toxin in different mycobacterial hosts}

The development of vaccines based on expression of recombinant antigens in $M$. bovis BCG (rBCG) is an ongoing area of research in numerous laboratories worldwide (Ohara \& Yamada, 2001; Nascimento et al., 2000; Leung et al., 2000; Langranderie et. al., 1998). Due to the extended growth time of BCG, a commonly used preliminary step in the analysis of test constructs involves the transformation of M. smegmatis $\mathrm{mc}^{2} 155$ to confirm antigen expression and evaluate vector stability in a mycobacterial environment (O'Gaora, 1997). To determine if $M$. vaccae could also be used for this purpose, we prepared two constructs, pPB10 and pPB12, each containing a sequence encoding the entire S1 subunit of pertussis toxin, which has been proposed as a candidate antigen in rBCG whooping cough vaccines (Abomoelak et al., 1999; Nascimento et al., 2000). These vectors were used to transform the three mycobacterial cloning hosts and duplicate cultures of each recombinant were processed and analysed by Western blotting. Expression of a strongly immunoreactive band with a molecular mass of $31.7 \mathrm{kDa}$ (Fig. 2) in combination with the presence of a second immunoreactive 
band with a molecular mass of $28.7 \mathrm{kDa}$ was observed in all pPB10 and pPB12 recombinants but was absent in control recombinants transformed with pUS973 or pUS977 (Fig. 2). The smaller band showed the same mobility as the S1 subunit control and as such is likely to represent mature S1 protein. The larger band is thought to represent a pro-form of the S1 subunit. The levels of antigen expression were equivalent in the three species and were estimated to be around 40 ng per $10^{6}$ cells, a value obtained based on comparison of the band intensities produced from known quantities of pertussis toxin which were included in all blots. This result was somewhat surprising given that the two expression vectors contained promoters that differed substantially in terms of their ability to drive the expression of recombinant $\beta$-galactosidase.

It should be emphasized that the monoclonal antibody 3CX4PT has been shown to confer passive immunity in mice challenged with pertussis toxin (Sato et al., 1991). Thus the reactivity of the two forms of recombinant $\mathrm{S} 1$ protein expressed by the three mycobacteria with this monoclonal antibody indicates the presence of serum neutralizing epitopes in the recombinant antigen.

\section{Analysis of vector stability}

The issue of vector stability, both structural and functional, within mycobacteria has received relatively little attention hitherto but has begun to achieve greater prominence particularly in the light of its implications for vaccine development.

Plasmid DNA was recovered from mycobacterial transformants as described in Methods and was used to transform E. coli $\mathrm{DH} 5 \alpha$ by electroporation. Vector DNA was purified and subjected to restriction digestion with BamHI, HindIII and EcoRI. The resulting restriction patterns were compared with those of the vectors originally used to transform the mycobacterial hosts. Gross modifications in vector structure were observed in $\mathrm{mc}^{2} 155$ (pUS973, 985, 989, 995, pPB10 and pPB12) and in M. vaccae (pUS972 and pUS985). In the majority of cases the modifications were represented by deletions but insertions were occasionally encountered.

A more detailed analysis of this phenomenon was performed using the vectors pUS985 and pUS989. The pUS985 construct was found to be highly unstable in $\mathrm{mc}^{2} 155$ with $90 \%$ of clones losing the ability to express $\beta$-galactosidase within two subcultures in liquid medium containing kanamycin. All of these clones were subsequently found to contain structurally modified vectors (Fig. 3). Thirty percent (3 of 10) of M. vaccae clones transformed with this vector were found to suffer structural modifications (Fig. 3) and loss of $\beta$-galactosidase expression within three subcultures. In contrast, the pUS989 vector was observed to be more stable in M. smegmatis $\mathrm{mc}^{2} 155$ with only one clone out of 10 suffering gross structural modifications over three subcultures. However, $40 \%$ (4 of 10) of the clones lost the ability to express $\beta$-galactosidase within three sub- cultures. In the case of M. vaccae, $20 \%$ (2 of 10 ) of the clones transformed with pUS989 lost the ability to express $\beta$-galactosidase but no evidence of gross structural modifications was detected. Finally, six individual E. coli clones transformed with either pUS985 or pUS989 were subcultured 10 times and no evidence of gross modifications was found. The ability to allow stable maintenance and expression of heterologous DNA is an essential feature of a cloning host and M. vaccae appears to fulfil these criteria adequately. Indeed, data from this study indicate that $M$. vaccae is less likely to induce either structural or functional plasmid instability than M. smegmatis. However, this will only be confirmed through a more detailed examination of this topic, using a wider range of vectors.

Our evaluation of the structural changes was limited to restriction digest analysis of the recovered plasmids, and as such we are unable to provided detailed information on the molecular nature of these changes. However, the presence of both insertions and deletions were reported by other workers (Haeseleer, 1994; Garbe et al., 1994; Kumar et al., 1998; Chawla \& Das Gupta, 1999; De Smet et al., 1999), which would suggest that the changes were transposon-induced in both $\mathrm{mc}^{2} 155$ and M. vaccae. Studies are under way in our laboratory to define the precise nature of the observed structural modifications.

\section{Existing cloning hosts and vector systems have limitations}

Immunological and biochemical studies of subcellular components of the pathogenic mycobacteria have been hampered by the difficulty in producing sufficient contaminant-free quantities of these molecules directly from such slow-growing organisms (Harth et al., 1997; Horn et al., 1999). Conventional recombinant protein technology has been applied in an attempt to resolve this problem, but suffers from the fact that many mycobacterial proteins are not faithfully produced in common cloning hosts such as E. coli (Hermans \& de Bont, 1996; Herrmann et al., 1996). Consequently, expression systems for use with $M$. smegmatis were developed for the production of recombinant forms of mycobacterial proteins (Zhang et al., 1991; Harth et al., 1997; Triccas et al., 1998; De Smet et al., 1999), although this approach has not proved to be completely satisfactory. In this context, it was reported that the expression of M. tuberculosis superoxide dismutase in M. smegmatis $\mathrm{mc}^{2} 155$ generated hybrid enzymes, whilst expression in $M$. vaccae allowed the recovery of the recombinant enzyme apparently in the native form (Garbe et al., 1994). Moreover, production of recombinant $45 / 47 \mathrm{kDa}$ molecules (Apa) of M. tuberculosis by $M$. smegmatis resulted in antigen with a different mannosylation pattern and reduced capacity to stimulate $\mathrm{T}$ lymphocyte responses in comparison to that observed for the native antigen (Horn et al., 1999). These observations and those of the present study demonstrate that the expression of recombinant proteins in mycobacteria is influenced by a number of factors 


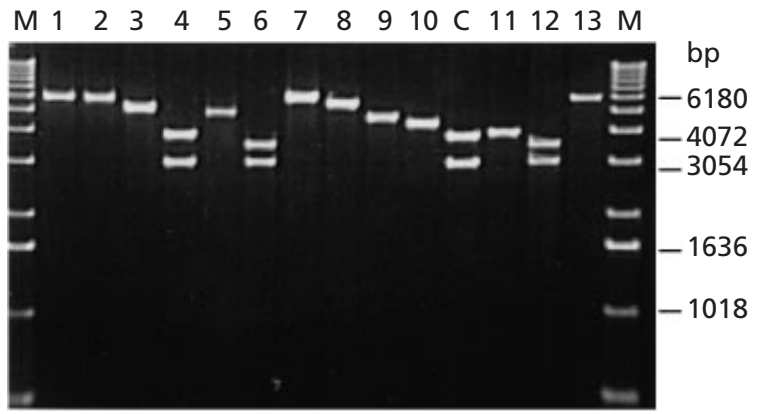

Fig. 3. Structural modifications in the vector pUS985 recovered from $M$. smegmatis $\mathrm{mc}^{2} 155$ and $M$. vaccae transformants. Lanes 1-10, plasmid pUS985 recovered from 10 individual clones of $M$. smegmatis $\mathrm{mc}^{2} 155$; lanes 11-13 plasmid pUS985 recovered from three individual clones of $M$. vaccae. C, control vector pUS985 purified from E. coli. All vectors were digested with HindIII. M, $1 \mathrm{~kb}$ ladder.

including vector structure, promoter strength and the nature of the cloned gene.

The identification of $M$. smegmatis $\mathrm{mc}^{2} 155$ as a surrogate cloning host represented a landmark in the study of mycobacterial genetics. The applications and properties of this strain have recently been reviewed (Jacobs, 2000), and it is beyond doubt that it has served as a powerful tool for the advancement of our knowledge in numerous areas of mycobacterial biology. Indeed, the successful use of $\mathrm{mc}^{2} 155$ virtually ended the search for alternative cloning hosts (Hermans \& de Bont, 1996). Yet, it has been argued by some authors (Garbe et al., 1994; Hermans \& de Bont, 1996) that although $M$. smegmatis $\mathrm{mc}^{2} 155$ is a useful model system for mycobacterial genetic studies, there exists a need for additional cloning hosts which may be more useful for specific aspects of mycobacterial biology and which would provide increased flexibility to the field of recombinant protein technology for mycobacteria. In this context $M$. vaccae, specifically ATCC 15483, represents an alternative mycobacterial cloning host that has been largely overlooked to date. We have determined that, in contrast to the findings of Garbe et al. (1994) using M. vaccae NCTC 11659, M. vaccae ATCC 15483 is capable of being transformed with a range of commonly used vectors that carry a gene encoding kanamycin resistance as a selectable marker. A comparison of M. vaccae ATCC 15483 with M. smegmatis $\mathrm{mc}^{2} 155$ as cloning hosts revealed that a range of characterized mycobacterial promoters cloned in the same promoter probe vector showed broadly similar activities, in terms of expression of the reporter enzyme $\beta$-galactosidase, in these two hosts and in BCG, demonstrating that existing expression systems can readily be employed with $M$. vaccae. These results were expanded through the observation that M. vaccae was capable of stable, in vitro expression of recombinant $\mathrm{S} 1$ subunit of pertussis toxin at levels equivalent to those seen with $\mathrm{rBCG}$ and $M$. smegmatis. Significantly, $M$. vaccae appears to offer the advantage of being less likely to induce either structural or functional plasmid instability than M. smegmatis, at least with the constructs examined in this study. Taken as a whole, these data clearly show that M. vaccae ATCC 15483 represents a versatile tool for use in the study of mycobacterial genetics.

\section{ACKNOWLEDGEMENTS}

This work was partially supported by grants from the PAPES 2 program of FIOCRUZ, the Wellcome Trust (047195/ Z/.96/Z) and the European Community (INCO-DC, ICA4CT-2000-30032). We are grateful to João Pedro Sousa Santos, LATER, Fiocruz for his help in the preparation of this manuscript.

\section{REFERENCES}

Abomoelak, B., Huygen, K., Kremer, L., Turnee, M. \& Locht, C. (1999). Humoral and cellular immune responses in mice immunized with recombinant Mycobacterium bovis Bacillus CalmetteGuérin producing a pertussis toxin-tetanus toxin hybrid protein. Infect Immun 67, 5100-5105.

Abou-Zeid, C., Gares, M. P., Inwald, J. \& 10 other authors (1997). Induction of a type- 1 immune response to a recombinant antigen from Mycobacterium tuberculosis expressed in Mycobacterium vaccae. Infect Immun 65, 1856-1862.

Chawla, M. \& Das Gupta, S. (1999). Transposition-induced structural instability of Escherichia coli-mycobacteria shuttle vectors. Plasmid 41, 135-140.

Clarke-Curtiss, J. E., Jacobs, W. R., Docherty, M. A., Ritchie, L. R. \& Curtiss, R., III (1985). Molecular analysis of DNA and construction of genomic libraries of Mycobacterium leprae. J Bacteriol 161, 1903-1102.

Corlan, E., Marica, C., Macavei, C., Stanford, J. L. \& Stanford, C. A. (1997). Immunotherapy with Mycobacterium vaccae in the treatment of tuberculosis in Romania. 2. Chronic or relapsed disease. Respir Med 91, 21-29.

Cormack, B.P., Valdivia, R. H. \& Falkow, S. (1996). FACS optimized mutants of green fluorescent protein (GFP). Gene 173, 33-38.

Das Gupta, S. K., Bashyam, M. D. \& Tyagi, A. K. (1993). Cloning and assessment of mycobacterial promoters by using a plasmid shuttle vector. J Bacteriol 175, 5186-5192.

Das Gupta, S., Jain, S., Kaushal, D. \& Tyagi, A. K. (1998). Expression systems for study of mycobacterial gene regulation and development of recombinant BCG vaccines. Biochem Biophys Res Commun 246, 797-804.

Dellagostin, O. A., Esposito, G., Earles, L.-J., Dale, J. W. \& McFadden, J. J. (1995). Activity of mycobacterial promoters during intracellular and extracellular growth. Microbiology 141, 1785-1792.

De Smet, K. A. L., Kempsell, K. E., Gallagher, A., Duncan, K. \& Young, D. B. (1999). Alteration of a single amino acid residue reverses fosfomycin resistance of recombinant MurA from Mycobacterium tuberculosis. Microbiology 145, 3177-3184.

Garbe, T. R., Barathi, J., Barnini, S., Zhang, Y., Abou-Zeid, C., Tang, D., Mukherjee, R. \& Young, D. B. (1994). Transformation of mycobacterial species using hygromycin resistance as a selectable marker. Microbiology 140, 133-138.

Golansaka, E., Brzostek, A., Kiatpapan, P. \& Dziadek, J. (1998). 
Characterisation of a new host-vector system for fast-growing mycobacteria. Acta Microbiol Pol 47, 335-343.

Haeseleer, F. (1994). Structural instability of recombinant plasmids in mycobacteria. Res Microbiol 145, 683-687.

Harth, G., Lee, B. Y. \& Horwitz, M. A. (1997). High-level heterologous expression and secretion in rapidly growing non-pathogenic mycobacteria of four major Mycobacterium tuberculosis extracellular proteins considered to be leading vaccine candidates and drug targets. Infect Immun 65, 2321-2328.

Hermans, J. \& de Bont, J. A. M. (1996). Techniques for genetic engineering in mycobacteria. Antonie Leeuwenhoek 69, 243-256.

Hermans, J., Boschloo, J. G. \& de Bont, J. A. M. (1990). Transformation of Mycobacterium aurum by electroporation: the use of glycine, lysozyme and isonicotinic acid hydrazide in enhancing transformation efficiency. FEMS Microbiol Lett 72, 221-224.

Hermans, J., Suy, I. M. L. \& de Bont, J. A. M. (1993). Transformation of Gram-positive microorganisms with the Gramnegative broad-host-range cosmid vector pJRD251. FEMS Microbiol Lett 108, 201-204.

Herrmann, J. L., O'Gaora, P., Gallagher, A., Thole, J. E. \& Young D. B. (1996). Bacterial glycoproteins: a link between glycosylation and proteolytic cleavage of a $19 \mathrm{kDa}$ antigen from Mycobacterium tuberculosis. EMBO J 15, 3457-3554.

Hetzel, C., Janssen, R., Ely, S. J., Kristensen, N. M., Bunting, K. Cooper, J. B., Lamb, J. R., Young, D. B. \& Thole, J. E. R. (1998). An epitope delivery system for use with recombinant mycobacteria. Infect Immun 66, 3643-3648.

Horn, C., Namane, A., Pescher, P., Rivière, M., Romain, F., Puzo, G., Barzu, O. \& Marchal, G. (1999). Decreased capacity of recombinant $45 / 47 \mathrm{kDa}$ molecules (Apa) of Mycobacterium tuberculosis to stimulate $\mathrm{T}$ lymphocyte responses related to changes in their mannosylation pattern. J Biol Chem 274, 32023-32030

Jacobs, W. R., Jr (2000). Mycobacterium tuberculosis: a once genetically intractable organism. In Molecular Genetics of Mycobacteria, pp. 1-16. Edited by G. F. Hatfull and W. R. Jacobs Jr. Washington, DC: American Society for Microbiology.

Kieser, T., Moss, T., Dale, J. W. \& Hopwood, D. A. (1986). Cloning and expression of Mycobacterium bovis DNA in 'Streptomyces lividans'. J Bacteriol 168, 72-80.

Kumar, D., Srivastava, B. S. \& Srivastava, R. (1998). Genetic rearrangements leading to disruption of heterologous gene expression in mycobacteria: an observation with Escherichia coli $\beta$-galactosidase in Mycobacterium smegmatis and its implication in vaccine development. Vaccine 16, 1212-1215.

Laemmli, U. K (1970). Cleavage of structural proteins during the assembly of the head of bacteriophage T4. Nature 227, 680-685.

Lagranderie, M., Winter, N., Balazuc, A. M., Gicquel, B. \& Gheorghiu, M. (1998). A cocktail of Mycobacterium bovis BCG recombinants expressing the SIV Nef, Env, and Gag antigens induces antibody and cytotoxic responses in mice vaccinated by different mucosal routes. AIDS Res Hum Retroviruses 18, 1625-1633.

Lazraq, R., Clavel-Seres, S. \& David, H. L. (1991). Transformation of distinct mycobacterial species by shuttle vectors derived from the Mycobacterium fortuitum pAL5000 plasmid. Curr Microbiol 22, 9-13.

Leung, N. J., Aldovini, A., Young, R. \& 7 other authors (2000). The kinetics of specific immune response in rhesus monkeys inoculated with live recombinant BCG expressing SIV gag, pol, env and nef proteins. Virology 268, 94-103.

Lim, E. M., Rauzier, J., Timm, J., Torrea, G., Murray, A., Gicquel, B.
\& Portnoi, D. (1995). Identification of Mycobacterium tuberculosis DNA sequences encoding exported proteins by using $p h o A$ gene fusions. J. Bacteriol 177, 59-65.

Mahenthiralingam, E., Draper, P., Davis, E. O. \& Colston, M. J. (1993). Cloning and sequencing of the gene which encodes the highly inducible acetamidase of Mycobacterium smegmatis. I Gen Microbiol 139, 575-583.

Miller, J. H. (1992). A Short Course in Bacterial Genetics: a Laboratory Manual and Handbook for Escherichia coli and Related Bacteria. Cold Spring Harbor, NY: Cold Spring Harbor Laboratory.

Murray, A., Winter, N., Lagranderie, M. \& 7 other authors (1992). Expression of Escherichia coli $\beta$-galactosidase in Mycobacterium bovis BCG using an expression system isolated from Mycobacterium paratuberculosis which induced humoral and cellular immune responses. Mol Microbiol 6, 3331-3342.

Nascimento, I. P., Dias, W. O., Mazzantini, R. P. \& 11 other authors (2000). Recombinant Mycobacterium bovis BCG expressing pertussis toxin subunit $\mathrm{S} 1$ induces protection against an intracerebral challenge with live Bordetella pertussis in mice. Infect Immun 68, 4877-4883.

O'Gaora, P. (1997). Expression of genes in mycobacteria. Methods Mol Microbiol 101, 261-274.

Ohara, N. \& Yamada, T. (2001). Recombinant BCG vaccines. Vaccine 19, 4089-4098.

Onyebujoh, P. C., Abdulmumini, T., Robinson, S., Rook, G. A. \& Stanford, J. L. (1995). Immunotherapy with Mycobacterium vaccae as an addition to chemotherapy for the treatment of pulmonary tuberculosis under difficult conditions in Africa. Respir Med 89, 199-207.

Parish, T. \& Stoker, N. G. (1997). Electroporation of mycobacteria. Methods Mol Microbiol 101, 129-144.

Parish, T., Mahenthiralingam, E., Draper, P., Davis, E. O. \& Colston, M. J. (1997). Regulation of the inducible acetamidase gene of Mycobacterium smegmatis. Microbiology 143, 22672276.

Pashley, C. \& Stoker N. G. (2000). Plasmids in mycobacteria. In Molecular Genetics of Mycobacteria, pp 55-67. Edited by G. F. Hatfull and W. R. Jacobs, Jr. Washington DC: American Society for Microbiology.

Santos, A. R., Miranda, A. B., Lima, L. M., Suffys, P. N. \& Degrave, W. M. (1992). Method for high yield preparation in large and small scale of nucleic acids from mycobacteria. J Microbiol Methods 15, 83-94.

Sato, H., Sato, Y. \& Ohishi, I. (1991). Comparison of pertussis toxin (PT)-neutralizing activities and mouse-protective activities of anti-PT mouse monoclonal antibodies. Infect Immun 59, 3832-3835.

Snapper, S. B., Melton, R. E., Mustaga, S., Kieser, T. \& Jacobs, W. R. (1990). Isolation and characterization of efficient plasmid transformation mutants of Mycobacterium smegmatis. Mol Microbiol 4, 1911-1919.

Stolt, P. \& Stoker, N. G. (1996). Functional definition of regions necessary for replication and incompatibility in the Mycobacterium fortuitum plasmid pAL5000. Microbiology 142, 27952802

Stover, C. K., de la Cruz, V. F., Fuerst, T. R. \& 11 other authors (1991). New use of BCG for recombinant vaccines. Nature 351, 456-460.

Timm, J., Lim, E. M. \& Gicquel, B. (1994). Escherichia coli-mycobacteria shuttle vectors for operon and gene fusions to lacZ: the pJEM series. J Bacteriol 176, 6749-6753. 
Towbin, H., Staehelin, R. \& Gordon, J. (1979). Electrophoretic transfer of proteins from polyacrylamide gels to nitrocellulose sheets: procedure and some applications. Proc Natl Acad Sci US A 76, 4350-4354.

Triccas, J. A., Parish, T., Britton, W. J. \& Gicquel, B. (1998). An inducible expression system permitting the efficient purification of recombinant antigens from Mycobacterium smegmatis. FEMS Microbiol Lett 167, 151-156.

Yasutomi, Y., Koenig, S., Haun, S. S. \& 7 other authors (1993). Immunization with recombinant BCG-SIV elicits SIV-specific cytotoxic $\mathrm{T}$ lymphocytes in rhesus monkeys. J Immunol 150, 3101-3107.

Zhang, Y., Lathigra, R., Garbe, T., Catty, D. \& Young, D. (1991). Genetic analysis of superoxide dismutase, the 23 kilodalton antigen of Mycobacterium tuberculosis. Mol Microbiol 5, 381391.

Received 17 October 2001; revised 11 March 2002; accepted 12 March 2002. 